\title{
The icosahedral 180 subunit capsid of the Aquifex aeolicus Lumazine synthase IDEA mutant dose not follow the quasi-equivalence
}

\author{
L. Xing*, J. Nilsson*, X.F. Zhang**, M.Fischer***, A.Bacher***, W. Meining**, R. Ladenstain**, and \\ R.H.Cheng* \\ * Department of Molecular and Cellular Biology, University of California at Davis, Davis, CA95616, \\ USA \\ ** Department of Biosciences at Novum, Karolinska Institute, 14157 Huddinge, Sweden \\ *** Lehrstuhl fur Organische Chemie und Biochemie, Technische Universitat Munchen, \\ Lichtenbergstr. 4, D-85747, Garching, Germany
}

Structure study by cryo-electron microscopy and image reconstructiuon has great power in monitoring biological dynamic events, such as virus cell entry and enzyme catalytic cycle. Lumazine synthase catalyzes the penultimate step in the biosynthesis of riboflavin (vitamin B), the universal precursor of flavocoenzyme. The enzyme exists broadly in plant and many microorganisms but animals must obtain it from dietary. The three-dimensional structure of several lumazine synthases have been studied by X-ray crystallography. However, the precise mechanism of the catalytic reaction cycle remained still unclear. Most existing functional and dynamic data of the enzyme are obtained from the studies on lumazine synthase from $B$ subtilis (LSBS) ${ }^{1}$. The LSBS is a capsid of 60 subunits arranged according to $\mathrm{T}=1$ icosahedral symmetry. The active site is located at the interface of two adjacent subunits in the close proximity to the inner surface of the icosahedral capsid ${ }^{2}$. The access of substrates to active site from outside environment is a dynamic process that involves capsid conformational changes and remains as a puzzle for decades. Here, we construct a LSBS mutant, in which four amino acids (Ile-Asp-GluAla) are inserted after Gly129 (LSBS-IDEA) and determined the structure of LSBS-IDEA by cryo-electron microscopy and image processing ${ }^{3}$. The pentamer implies us a confirmation which could be formed during enzyme catalytic cycle

The LSBS-IDEA assembles into two types of particles that projected into spherical images of different sizes on cryo-micrograph (Fig 1). The three-dimensional reconstruction representing the majority of the particles reveals an icosahedra capsid with diameter of $292 \AA$ (Fig 2), which is larger than native LSBS particle of $160 \AA$. The capsid is composed of 180 copies of subunits with three unique monomers per icosahedral asymmetric unit. The map rendered according to volume density reveals that monomer $\mathrm{B}$ and monomer $\mathrm{C}$ (around the pentamer ring) locate at different radial position (Fig 2, right panel). The IDEA loop structure from yeast enzyme ${ }^{4}$ was manually docked into LSBS coordinate and then the LSBS coordinates was modeled into cryo-EM density map. The final fitting gave 89\% similarity between EM density map and the map generated from coordinates (Fig 3). The fitting further demonstrates that the interactions among three unique subunits follow neither quasi-symmetry nor similarity to their geometrically equivalent components. Therefore, the 180 copies of LSBS-IDEA subunit violate the theory of icosahedral quasi-equivalence $^{5}$. The IDEA loop would introduce clash with adjacent pentamer if LSBSIDEA subunit remained its native LSBS orientation. To avoid such spatial hindrance, LSBSIDEA rotates $36^{\circ}$ along an axis perpendicular to its molecular long axis and translates $3 \AA$ away from icosahedral fivefold axis. Such movement results in pentamer expansion so that the diameter of fivefold central pore increases from $9 \AA$ to $19 \AA$ (Fig 3). Most strikingly, the movement alters the interface of two adjacent subunits from close tight contact to loose open 
conformation. Consistent to the structure data, LSBS-IDEA shows no enzymatic activity. Therefore, the expanded pentamer revealed here by LSBS-IDEA mutant indicates an alternative conformation of lumazine synthase when the substrates are absent.

\section{References:}

1. Fischer and Bacher (2005) Nat. Prod.Rep. 22:324. 2. Ladenstein et al., (1988) JBC 203: 198. 3. Baker and Cheng (1996) J. Struct. Biol. 116: 120. 4. Meining et al., (2000) JMB 299: 181. 5. Caspar and Klug (1962) Cold Spring Haror Symp. Quant. Biol. 27:1.

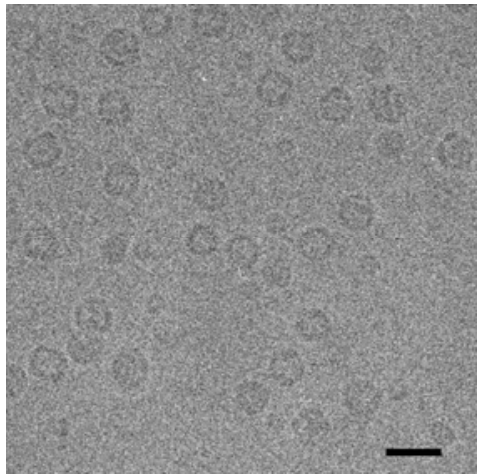

Figure 1: cryo-micrography of LSBS-IDEA mutant reveals two types of spherical ring like projections. The large projection is the majority about $70 \%$ in population. Bar $=10 \mathrm{~nm}$

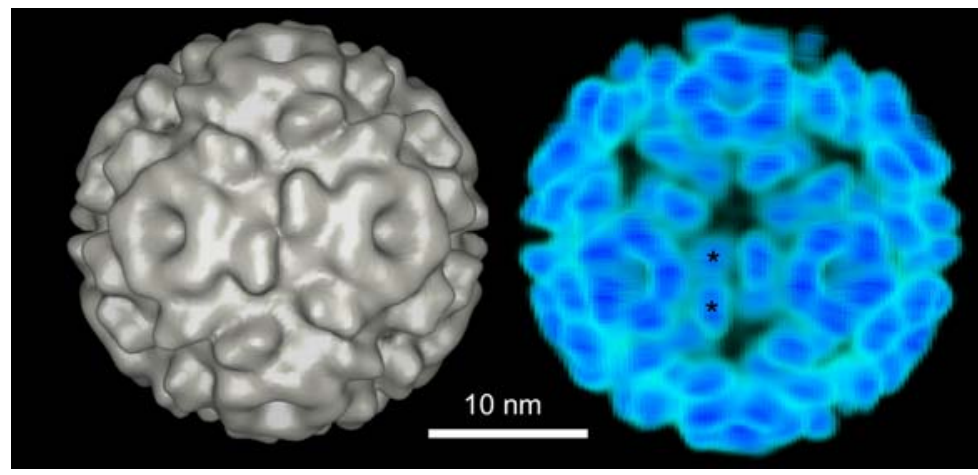

Figure 2: The three dimensional structure of LSBS-IDEA mutant presented as surface rendering (left) and as volume rendering (right). The volume rendering map reveals three subunits within an asymmetric unit and the subunits (marked as asterisks) around pentamer locate at different radial position.
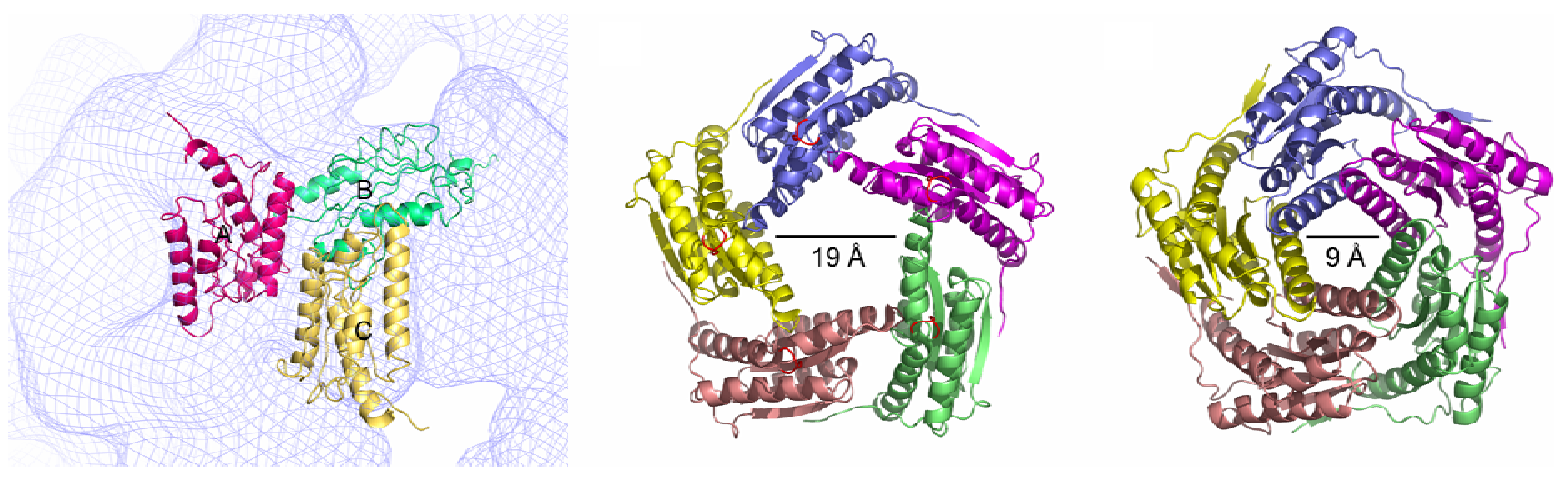

Figure 3: The coordinate of LSBS-IDEA (ribbon drawing) fits well to cryoEM density map (blue net) (left). The modeling reveals an expanded pentamer compared to native LSBS pentamer (right). LSBS-IDEA pentamer (middle) consists of a central pore of $19 \AA$ and open loose contact at enzyme active site. 